clinician-based collection for HR-HPV DNA testing, with 'good' agreement between both methods, high sensitivity (95.0\%; 95\%CI: $88.3-100.0 \%)$ and specificity $(88.2 \%$; 95\% CI: 83.9-92.6\%). Remarkably, the rates of HPV DNA and HR-HPV DNA positivity were significantly higher (1.67- and 1.57- fold, respectively) when using veil-based collection than clinician-collection.

Conclusion These observations highlight the unsuspected high burden of cervical oncogenic HR-HPV infection in Chadian women. Self-collection of genital secretions using the V-Veil-Up Gyn Collection Device constitutes a simple, highly acceptable and powerful tool to collect genital secretions for further molecular testing and screening of oncogenic HR-HPV that could be easily implemented in the national cervical cancer prevention program in Chad.

Disclosure No significant relationships.

\section{P825 HPV SEROPREVALENCE AND SEROCONVERSION AMONG HIV-POSITIVE MEN: COHORT STUDY IN SOUTH AFRICA}

${ }^{1}$ Admire Chikandiwa*, ${ }^{2}$ Helena Faust, ${ }^{3}$ Philippe Mayaud, ${ }^{2}$ Joakim Dillner, ${ }^{1}$ Sinead DelanyMoretlwe. 'Wits RHI, University of the Witwatersrand, Johannesburg, South Africa; ${ }^{2}$ Karolinksa Institute, Stockholm, Sweden; ${ }^{3}$ LSHTM, UK

10.1136/sextrans-2019-sti.870

Background The HPV seropositivity following natural infection can provide data on cumulative exposure to HPV. Studies evaluating seropositivity prospectively among men living with HIV (MLHIV) are few. We aimed to determine HPV type specific seroprevalence and seroconversion among MLHIV following natural HPV infection.

Methods We enrolled 304 sexually-active MLHIV $\geq 18$ years from Johannesburg. We collected socio-behavioral data, blood (CD4+ counts, HIV-1 plasma viral load [PVL] and serology), and genital swabs (HPV genotyping with Roche Linear Array and HPV 16/18 Viral Load [VL]) at enrolment and 6-monthly follow-up visits for up to 18 months. At enrolment and 18 months later, type-specific serum antibodies to $15 \mathrm{HPV}$ types (HPV6/11/16/18/31/33/35/39/45/52/56/58/59/68/73) were measured using HPV pseudovirions. Logistic regression evaluated factors associated with HPV seroconversion.

Results At enrolment, median age was 38 (IQR: 22-59) years, $25 \%$ reported $\geq 1$ sexual partners in the past 3 months and $5 \%$ reported ever having sex with other men. Most participants (65\%) were on ART, with median CD4+ count 445 cells/ $\mu \mathrm{L}$ (IQR: 328-567). Serology results were available for $99 \%$ of the 304 and 257 men who completed enrolment and 18 months visits. Seroprevalence of any HPV type was $66 \%$. Seropositivity for any HPV types of the bivalent (HPV16/18), quadrivalent (HPV6/11/16/18) and nonavalent (HPV6/11/16/ $1831 / 33 / 45 / 52 / 58)$ vaccines were $19 \%, 37 \%$ and $60 \%$ respectively. Among 59 men with genital HPV-DNA but seronegative for the same type at enrolment, 12 (22\%) had type-specific seroconversion at month 18 . Among these men, the risk of type specific seroconversion was higher among men with detectable PVL $(\mathrm{aOR}=2.78$, 95\%CI: 1.12-6.77), and HPV 18 $\mathrm{VL} \geq 5.3 \log 10 / 106$ cells ( $\mathrm{aOR}=3.32$, 95\%CI: 1.42-7.74).
Conclusion MLHIV have high HPV seroprevalence rates implying that prevention of HPV infection is required. There is evidence of seroconversion in response to detectable DNA infection at baseline, which was associated with both high HIV and HPV 18 viral loads.

Disclosure No significant relationships.

\section{P826 HIGH PREVALENCE OF CERVICAL HR-HPV IN IMMIGRANT WOMEN ORIGINATING FROM SUB- SAHARAN AFRICA AND LIVING IN ORLÉANS, FRANCE}

${ }^{1}$ Ralph Sydney Mboumba Bouassa*, ${ }^{2}$ Camelia Gubavu, ${ }^{3}$ Mathieu Matta, ${ }^{3}$ Leman Robin, ${ }^{3}$ David Veyer, ${ }^{2}$ Anne Gravier, ${ }^{2}$ Laurent Hocqueloux, ${ }^{2}$ Thierry Prazuck, ${ }^{3}$ Hélène Péré, ${ }^{3}$ Laurent Bélec. ${ }^{1} E$ Ecole Doctorale d'Infectiologie Tropicale, Tropicale Infectiologie, Franceville, Gabon; ${ }^{2}$ Service des Maladies Infectieuses et Tropicales, Centre Hospitalier Régional d'Orléans, Orléans, France; ${ }^{3}$ Laboratoire de Virologie, Hôpital Européen Georges Pompidou and Faculté de Médecine Paris Descartes, Université Paris Descartes (Paris V), Sorbonne Paris Cité, Paris, France

\subsection{6/sextrans-2019-sti.871}

Background We herein assess the HPV detection and HPVrelated cervical lesions in immigrant African women living in France.

Methods In 2018, immigrant African adult women attending for sexual health and HIV care a French healthcare facility, were included and subjected to endocervical swabs and Pap smear. HPV DNA was detected by multiplex real-time PCR (AnyplexTM II HPV28, Seegene, Seoul, South Korea).

Results 50 African immigrant women (mean age, 41.7) residing in France for an average of 10.7 years (range 1-32), including $74.0 \%$ of HIV-positive and $26.0 \%$ of HIV-negative, were prospectively included. HPV prevalence was $68.0 \%$, with $56.0 \%$ of HR-HPV and $24.0 \%$ of multiple HPV. Unexpectedly, the non-vaccine HR-HPV-68 (20.0\%) was the predominant genotype, followed by the Gardasil- $9^{\oplus}$ vaccine HR-HPV-58 (14.0\%). HPV-16 and HPV-18 were respectively detected in $6.0 \%$ and $10.0 \%$. HIV-infected women were more infected with HR-HPV (62.2\%) and multiple HR-HPV (16.2\%) than HIV-negative women (38.4\% and 7.7\%, respectively), $(\mathrm{P}=0.19$ and $\mathrm{P}=0.66$, respectively). Most women (84.0\%) showed normal cytology, but $16.0 \%$ exhibited cervical abnormalities, including $6.0 \%$ of Low-grade Squamous Intraepithelial Lesions (LSIL) and Abnormal Squamous Cells with Unknown Significance (ASCUS), and 4.0\% of High-grade Squamous Intraepithelial Lesions (HSIL). HPV-16 and HPV-18 were detected in one woman with HSIL. Finally, $87.5 \%$ of the study women with cervical abnormalities were HIV-positive, while only one HIV-negative woman showing cervical abnormalities.

Conclusion These findings highlight the unsuspected high burden of cervical HR-HPV infections often associated with cervical abnormalities in the first-generation immigrant women originating from sub-Saharan Africa and residing in France. These observations also point out the atypical epidemiological profile of cervical HPV infection with a rare and non-vaccine high-risk genotype being the most detected HPV, raising thus a concern about the low predictive efficacy of the Gardasil- $9^{\text {क }}$ vaccine in the prevention of the main genotypes circulating in these specific population residing in France.

Disclosure No significant relationships. 